\title{
Ultrasonography used in the diagnosis of chronic rhinosinusitis: From experimental imaging to clinical practice
}

\author{
ADRIANA NEAGOS ${ }^{1,2}$, MIHAI DUMITRU ${ }^{3}$, DANIELA VRINCEANU ${ }^{4}$, \\ ADRIAN COSTACHE ${ }^{5}$, ANDREEA NICOLETA MARINESCU ${ }^{6}$ and ROMICA CERGAN ${ }^{3}$
}

\begin{abstract}
${ }^{1}$ ENT Department, 'George Emil Palade' University of Medicine, Pharmacy, Science and Technology of Targu Mures, 540139 Târgu Mureș; ${ }^{2}$ Galenus Medical Center, 540342 Targu Mures; ${ }^{3}$ Department of Anatomy, 'Carol Davila' University of Medicine and Pharmacy, 050474 Bucharest; ${ }^{4}$ ENT Department, Bucharest Emergency University Hospital, 010271 Bucharest; ${ }^{5}$ Department of Pathology, 'Carol Davila' University of Medicine and Pharmacy, 020021 Bucharest; ${ }^{6}$ Department of Radiology and Medical Imaging, Bucharest Emergency University Hospital, 030171 Bucharest, Romania
\end{abstract}

Received December 31, 2020; Accepted February 2, 2021

DOI: $10.3892 /$ etm.2021.10043

\begin{abstract}
There is an increasing incidence of sinusitis cases in outpatient clinics; therefore, new methods for screening and triage are required. Performance of sinus sonography in our outpatient protocol was assessed in order to ascertain the specificity and sensitivity of this imaging method to detect rhinosinusitis. A total of 81 consecutive cases with clinical signs of sinusitis were collected and clinical data compared with sinus sonography results. Moreover, sinus sonography enhanced referral for further computed tomography scans. The results showed that sonography may be a screening method in sinus pathology, with a high sensitivity of $78.3 \%$ and a specificity of $93.1 \%$. In the context of the COVID-19 pandemic, the use of ultrasound for paranasal sinus imaging should be reconsidered as in the case of lung sonography. According to data of the present study, sinus ultrasound may be implemented in the emergency departments with no access to standard ENT services and it could be efficient in managing sinusitis in pregnant women and pediatric cases.
\end{abstract}

\section{Introduction}

Heidelbach and Gilbricht were among the first to report the use of A-mode sonography for the analysis of maxillary sinusitis cases, which was later used broadly due to the enhancement of probes and because it represented a viable alternative to standard radiology (1).

Evidently, the gold standard in sinus imaging are computed tomography (CT) and magnetic resonance imaging and because

Correspondence to: Dr Daniela Vrinceanu, ENT Consultant, ENT Department, Bucharest Emergency University Hospital, Splaiul Independentei 169, 010271 Bucharest, Romania

E-mail: vrinceanudana@yahoo.com

Key words: ultrasound, sinus, rhinosinusitis, sensitivity, specificity of their development the use of sonography was abandoned until recently, when it was used in emergency departments in trauma cases for rapid detection of hemosinus (2).

Promising results were obtained in the management of maxillary acute sinusitis in pediatric cases given the advantage of reducing child exposure to radiation and enabling the serial exam during treatment (3). Therefore, ultrasonography can be used in pregnant patients to exclude acute sinusitis requiring active medical treatment precarious to pregnancy.

After 2005, the increased accessibility to high resolution ultrasound machines that were implemented aimed to analyze the specificity and sensitivity of this method for the detection of sinus fractures compared to standard radiology and CT scans. According to Adeyemo and Akadiri, the sensitivity and specificity for detecting orbital fractures was between 56-100 and 85-100\%, respectively; for the detection of nasal fractures, they recorded $90-100 \%$ sensitivity and $98-100 \%$ specificity compared to CT scans (4).

For the present study, we implemented the ultrasound exam of patients with inflammatory sinus pathology before they received CT scans in order to prove the utility of sonography in the primary management of these cases and in deciding for further referral to CT scan imaging.

\section{Materials and methods}

General. A total of 81 cases (48 males and 33 females), with a median age of 56 years, with sinus symptoms presented to a private outpatient clinic (Galenus Medical Center, Targu Mures, Romania) and were enrolled in the present study. Inclusion signs and symptoms: Pus secretion in the nasal cavity, mild facial pain, pressure sensation on sinus points, nasal obstruction, loss of smell, fever, headache.

All cases received standard ENT exam with nasal endoscopy, followed by sonography with an Acuson x300 machine. Following these steps, some of the cases were referred to CT scan imaging.

The study followed the international regulations in accordance with the Declaration of Helsinki. The study was approved by the Ethics Committee of Galenus Medical Center. 
Patient informed consent for publication of the data/images associated with the manuscript was obtained.

Method. The technique for ultrasound exam of sinuses is based on two important aspects: Precise delineation of bony structures in sonography window through the back-shadow effect, and the fact that, in pneumatic cavities, the ultrasound trajectory is reduced without a contiguous medium.

At the level of the frontal sinus, the transducer is placed over the upper rim of the orbit centered on the median line. Therefore, one can visualize the anterior wall of the sinus and the posterior echo generated through the encounter of ultrasound rays with the posterior sinus wall. In the case of acute sinusitis, the fluid content filling the sinus will enable the contiguous propagation of the ultrasound rays between the two walls. Sinus sonography is performed with the patient sitting upright and facing downwards. Therefore, the content of the sinus is moved downwards on the anterior wall and can be measured in quantity. Subsequently, the patient is examined with the head upright and if the content moves inferiorly, it will confirm the suspicion of a frontal purulent sinusitis. The absence of this movement will raise the suspicion of a cyst and even a sinus tumor. These steps are shown in Fig. 1.

Moreover, we examined the integrity of the nasal bone pyramid while angling the transducer over the lateral aspect obliquely to the inner angle of the eye. The structures visualized are nasal bones with posterior shadow effect and alar cartilages.

Imaging of the maxillary sinus is similar to that of the frontal sinus following examination of the integrity of the anterior sinus wall and the posterior back echo generated at the level of the posterior wall of the sinus. There are limits in the fact that cystic pathology on the posterior wall is hard to visualize.

The case of a patient with polypoid maxillary mucosa on the right side is presented. We enhanced the ultrasound exam protocol by visualizing both sinuses in duplex comparative window (Fig. 2).

In the duplex ultrasound window, the difference in thickness of the sinus mucosa on the anterior right maxillary wall compared to the thinner one on the anterior left maxillary wall is underlined. Due to the presence of the content, we obtained an important posterior echo on the right side, but on the left side, the normal sinus content had an absent posterior echo.

Statistical analysis. Statistical analysis was performed with Microsoft Excel Program using the Chi square test, and Spearman's correlation for P-values $<0.05$. In order to ascertain the performance of sonography as a screening test, we used the ROC curve therefore calculating the sensitivity and specificity.

\section{Results}

Patients. The median age of the study subjects was 56 years. The male predominance was of 59.25\%. The ultrasound exam compared to nasal endoscopy presented $78.3 \%$ sensitivity and $93.1 \%$ specificity. Only $28.39 \%$ of the patients were further referred to CT scan imaging. Two of the 81 patients proved to be suffering from mucocele and sinus tumor.

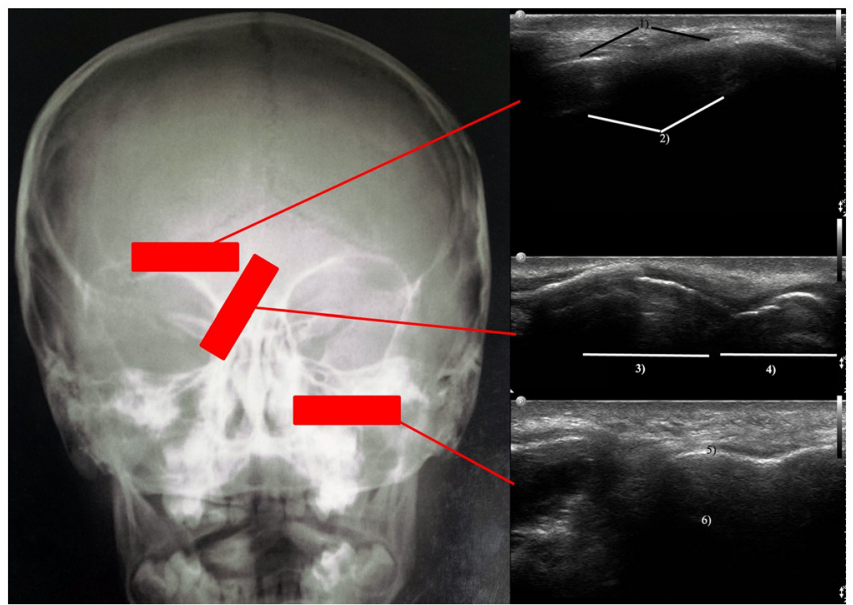

Figure 1. Sinus ultrasound protocol: Left image the transducer positioning, upper right image at the level of the frontal sinus; middle right image at the level of the lateral aspect of the nasal pyramid; lower right image at the level of the maxillary sinus. Therefore, one can visualize the anterior wall of the sinus 1) and the posterior echo generated through the encounter of ultrasound rays with the posterior sinus wall 2). The structures visualized are nasal bones 3 ) with posterior shadow effect and alar cartilages 4). In addition, the integrity of the anterior sinus wall 5) and the posterior back echo generated at the level of the posterior wall of the sinus 6) are visualized.

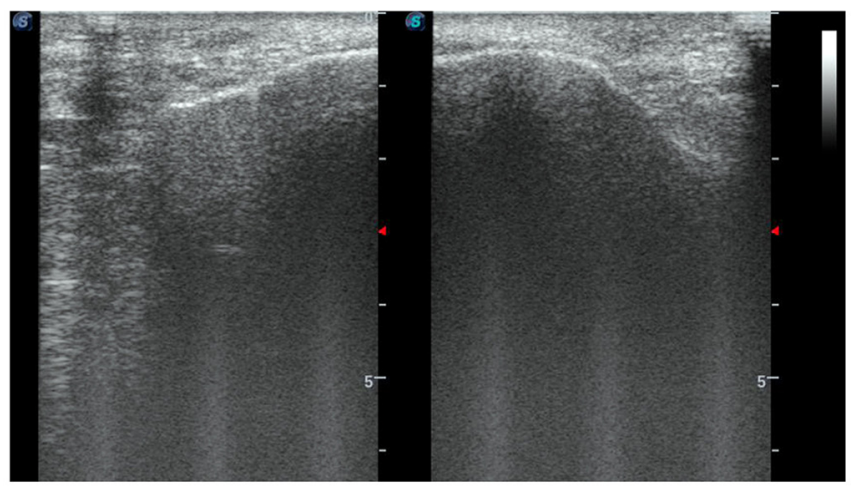

Figure 2. Duplex sonography of both maxillary sinuses in the same patient.

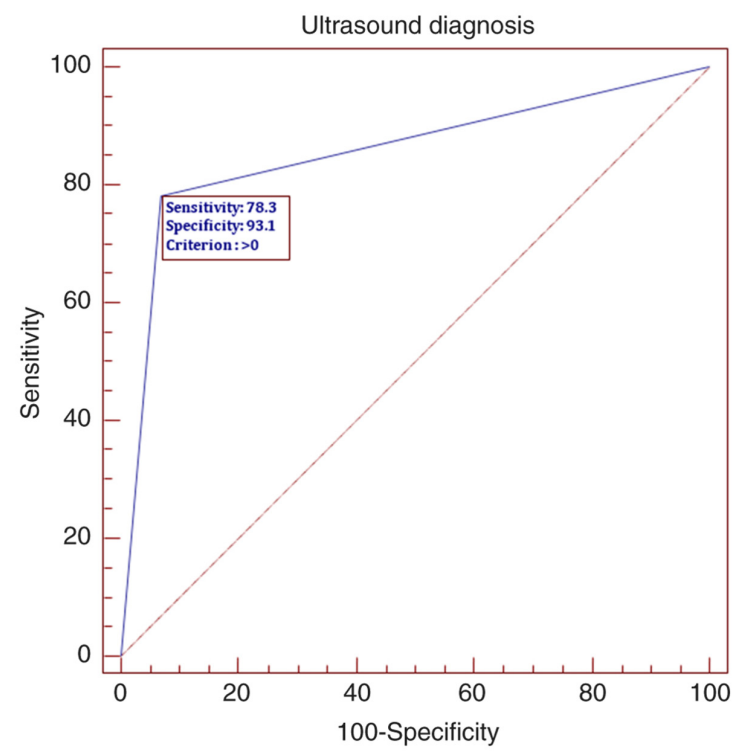

Figure 3. ROC curve for sinus ultrasound revealing sensitivity of 78.3 and $93.1 \%$ specificity. 
Spearman's correlation. The age group distribution revealed a greater incidence of sinus pathology of $27.16 \%$ between 41 and 50 years of age.

In addition, the ultrasound exam suggested the diagnosis of rhinosinusitis in $28.39 \%$ of the cases. We obtained a statistical P-value of 0.002 for the correlation between sex and age groups, with a lower incidence of male patients $<40$ years and a female predominance between 41 and 50 years. Regarding the correlation between clinical diagnosis and age groups, we did not obtain a positive statistical result. However, we obtained a statistical P-value of 0.04 for correlating ultrasound diagnosis with age groups. Furthermore, there was an increased incidence of ultrasound diagnosis of rhinosinusitis is in the age groups 41-50 and 71-80 years. Moreover, in the age group of 51 to 60 years, the ultrasound confirmed the clinical suspicion of chronic rhinosinusitis. While correlating the sex of the patients with the ultrasound diagnosis we obtained a P-value of 0.01 ; in 40 male cases, the ultrasound confirmed the clinical diagnosis of rhinosinusitis. Further correlation between ultrasound imaging and further referral to CT scan retrieved a significant P-value of 0.0001 .

By plotting the ROC curve, we established that sonography may be a screening method in sinus pathology with a high sensitivity of 78.3 and $93.1 \%$ specificity, (Fig. 3).

\section{Discussion}

Data obtained thus far prove the utility in screening pediatric population or in emergency departments. There were also studies that analyzed the utility of sonography in imaging the bone sections performed during rhinoplasty procedures and for correct visualization of the repositioned bone fragments in trauma cases $(5,6)$.

The increased incidence of cranial and facial trauma requires the development of new screening protocols in the emergency departments to expedite the access of major cases to complex imaging studies (7). This is also the case for patients with sinusitis complications in which the treatment progression could be actively followed using ultrasound (8). Ultrasonography could be the answer to these problems, as it attracts more ENT specialists towards its use in outpatient settings $(9,10)$.

Moreover, there is a current trend in surgeon-performed sonography, as in the case of gastrointestinal pathology, and this could also be applied in sinus pathology lowering the burden in already crowded imaging departments (11). Our sensitivity and specificity data are consistent with the ones published on 74 patients by Hsu et al (12).

Correlating allergic rhinitis cases with nasal sinus ultrasound could be a future step in the management of this pathology (13). Thus, we hope that our data will help shift the use of sonography from experimental to clinical practice in ENT and other allied specialties, such as ophthalmology, plastic surgery, and trauma surgery $(14,15)$.

However, there is the drawback of receiving incomplete data regarding nasal anatomy via this method, data that are necessary for proper planning of functional endoscopic sinus surgery.

In summary, sonography is an imaging method that has many advantages: Lack of irradiation, it is fast and cheap, and it permits serial dynamic examinations to ascertain the treatment efficacy. In the present study, we have shown that, in outpatient settings, sinus ultrasound has high sensitivity and specificity in ruling out cases without rhinosinusitis.

\section{Acknowledgements}

Professional editing, linguistic and technical assistance performed by Irina Radu, Individual Service Provider, certified translator in Medicine and Pharmacy (certificate credentials: Series E no. 0048).

\section{Funding}

No funding was received.

\section{Availability of data and material}

All data generated or analyzed during this study are included in this published article.

\section{Authors' contributions}

AN and MD contributed substantially to the conception and design of the study, the acquisition, analysis, and interpretation of the data, and were involved in the drafting of the manuscript. DV and AC contributed substantially to the analysis and interpretation of the data and were involved in the drafting of the manuscript. ANM and RC contributed substantially to the interpretation of the data and were involved in the critical revisions of the manuscript for important intellectual content. All authors agreed to be accountable for all aspects of the work in ensuring that questions related to the accuracy or integrity of any part of the work are appropriately investigated and resolved. All authors read and approved the final version of the manuscript.

\section{Ethics approval and consent to participate}

The study followed the international regulations in accordance with the Declaration of Helsinki. The study was approved by the Ethics Committee of Galenus Medical Center. Patient informed consent for publication of the data/images associated with the manuscript was obtained.

\section{Patient consent for publication}

Not applicable.

\section{Competing interests}

The authors declare that they have no competing interests.

\section{References}

1. Heidelbach JG and Gilbricht E: Practical experience in ultrasonic diagnosis of the great paranasal sinuses and their bases. Z Arztl Fortbild (Jena) 66: 208-210, 1972 (In German).

2. Bektas F, Soyuncu S and Yigit O: Acute maxillary sinusitis detected by bedside emergency department ultrasonography. Int J Emerg Med 3: 497-498, 2010. 
3. Puhakka T, Haikkinen T, Makela MJ, Alanen A, Kallio T, Korsoff L, Suonpää J and Ruuskanen O: Validity of ultrasonography in diagnosis of acute maxillary sinusitis. Arch Otolaryngol Head Neck Surg 126: 1482-1486, 2000.

4. Adeyemo WL and Akadiri OA: A systematic review of the diagnostic role of ultrasonography in maxillofacial fractures. Int J Oral Maxillo Surg 40: 655-661, 2011.

5. Abu-Samra M, Selmi G, Mansy H and Agha M: Role of intra-operative ultrasound-guided reduction of nasal bone fracture in patient satisfaction and patient nasal profile (a randomized clinical trial). Eur Arch Otorhinolaryngol 268: 541-546, 2011.

6. Anghel I, Anghel AG, Soreanu CC and Dumitru M: Craniofacial trauma produced by a violent mechanism. Coltea ENT Clinic experience. Rom J Leg Med 20: 215-218, 2012.

7. Enache G, Rusu E, Ilinca A, Rusu F, Costache A, Jinga M, Pănuş C and Radulian G: Prevalence of overweight and obesity in a Roma Population from southern Romania-Calarasi county. Acta Endocrinol (Buchar) 14: 122-130, 2018

8. Rusu E, Jinga M, Rusu F, Ciurtin C, Enache G, Dragomir A, Cristescu V, Stoica V, Costache A, Cheta D, et al: Statin therapy in patients with diabetes and hepatitis C. Farmacia 61: 1204-1215, 2013.

9. Costache A, Dumitru M, Anghel I, Cergan R, Anghel AG and Sarafoleanu C: Ultrasonographic anatomy of head and neck-a pictorial for the ENT specialist. Med Ultrason 17: 104-108, 2015.
10. Costache A, Dumitru M, Tweedie D, Sarafoleanu C and Anghel I: Adult cervical lymphangioma-ultrasonography, surgical removal, and pathology results. Case report. Med Ultrason 17: 411-413, 2015.

11. Georgescu EF, Mogoantă SȘ, Costache A, Pârvănescu V, Totolici BD, Pătraşcu S and Stănescu C: The assessment of matrix metalloproteinase-9 expression and angiogenesis in colorectal cancer. Rom J Morphol Embryol 56: 1137-1144, 2015.

12. Hsu CC, Sheng C and Ho CY: Efficacy of sinus ultrasound in diagnosis of acute and subacute maxillary sinusitis. J Chin Med Assoc 81: 898-904, 2018.

13. Berghi NO, Dumitru M, Vrinceanu D, Ciuluvica RC, SimioniucPetrescu A, Caragheorgheopol R, Tucureanu C, Cornateanu SR and Giurcaneanu C: Relationship between chemokines and $\mathrm{T}$ lymphocytes in the context of respiratory allergies (review). Exp Ther Med 20: 2352-2360, 2020.

14. Gradinaru S, Popescu LM, Piticescu RM, Zurac S, Ciuluvica R, Burlacu A, Tutuianu R, Valsan SN, Motoc AM and Voinea LM: Repair of the orbital wall fractures in rabbit animal model using nanostructured hydroxyapatite-based implant. Nanomaterials (Basel) 6: 11, 2016.

15. Cherecheanu PA, Istrate S, Iancu R, Popescu M, Bastian A and Ciuluvica R: Nanostructured hydroxyapatite used as an augmenting material to expand the orbit. Acta Ophthalmol: 95, 2017. 